\title{
ADJUVANT CHEMOTHERAPY FOR TESTICULAR TERATOMA WITH BLEOMYCIN AND VINBLASTINE
}

\author{
LT COL IR HAYWOOD, FRCS, RAMC \\ British Military Hospital, Munster \\ DR CLARE R TOPHAM, FRCR \\ St. Luke's Hospital, Guildford \\ DR F J F MADDEN, MRCP, FRCR \\ Leicester Royal Infirmary
}

SUMMARY: Nine patients with Stage I and II testicular teratoma received adjuvant chemotherapy with Bleomycin and Vinblastine commenced after the completion of conventional postoperative radiotherapy. Three have died with short disease free intervals. It is concluded there may be a case for adjuvant therapy, and in some cases possibly this should be commenced prior to radiotherapy.

\section{Introduction}

Adjuvant chemotherapy is now being employed in the management of several solid tumour malignancies in an attempt to improve prognosis. It is based on the concept that chemotherapy has the greatest chance of eliminating the disease when the tumour cell mass is minimal, and therefore, it is argued that if chemotherapy be employed at a stage when metastases are likely to be present, but as yet have not become apparent (micro-metastases), they may be eliminated completely in a proportion of cases. Many centres report work based on this hypothesis, notably Bonnadonna et al and Fischer with breast cancers, and currently trials are in progress with other solid neoplasms such as bone tumours with encouraging results. We are endeavouring to extend this work to Stage I and Stage II Testicular Teratoma and we publish a preliminary report on nine such cases.

Testicular teratoma is a disease mainly of males in their third and fourth decades, and not surprisingly is one of the commoner maligancies seen in service personnel, especially during years of conscription. Some of the larger series in this disease have come from military sources both in the United States and Great Britain. The British Army experience has been extensively reviewed in the past, notably, by Stephen ${ }^{1}$ on clinical aspects and Field ${ }^{2}$ on pathology.

The chemosensitivity of these tumours has been shown by the high remission rates achieved in many centres in the treatment of disseminated disease, the most recent being a rate of $80 \%$ of complete remission using vinblastine, Bleomycin and Cisplatin reported by Einhorn ${ }^{3}$. Encouraged by the results of Samuels ${ }^{4}$, we selected an adjuvant regime of Bleomycin and vinblastine, but one patient received vincristine alone.

The prognosis of these neoplasms depends upon the extent of the disease at presentation, histological type, and time between presentation and treatment (Field ${ }^{5}$ ).

\section{Staging}

Many staging profiles have been recommended and these tend to divide into 
North American and European groups. For simplicity our patients were staged as recommended by Staubitz ${ }^{6}$, namely :-

Stage I-disease confined to the Testes. Stage II-metastatic spread to the retro-peritoneal nodes. Stage III-further distant spread.

Stage II was assessed essentially by lymphography. It has not been our policy to carry out surgical excision of lymph nodes neither for treatment nor for staging biopsy, and, thus, we were unable to sub-divide our Stage I patients into those with or without microscopic metastases in the nodes as recommended by Maier ${ }^{2}$.

\section{Histological type}

Histological type, of which there are many, affects prognosis considerably but unfortunately the concensus amongst histopathologists as to the significance of var. ied features of the types is far from uniform. The histology of all cases has been reviewed and although initially classified according to the English classification as recommended by the British Testicular Tumour Panel $\left(\mathrm{Pugh}^{8}\right)$, they have been reclassified in addition according to the recommendations of Parkinson?

The British panels classification simplified, considered teratoma to be divided as :- (a) Differentiated (TD). (b) Intermittently differentiated (MTI), in other words, contained some differentiated tissue. (d) Totally undifferentiated (MTU). (e) Tumours consisting of trophoblastic tissue (MTT).

Parkinson and Beilby suggested in addition to the established principle that the presence of trophoblastic elements in a mixed tumour indicates a poor prognosis, the presence of yolk sac elements (endodermal sinus) which like trophoblast are of extra-embryonic origin, should be considered likewise. They further suggest that in a large number of tumours, tissue previously considered to be undifferentiated is probably of yolk sac origin. This tissue is possibly the source of Alpha-feto-protein secreted by some tumours. Prognosis then depends upon the presence of extraembryonic tissue, and in the case of yolk sac tissue apparently it is proportional to the quantity. The presence of somatic elements, irrespective of their degree of differentiation, does not affect prognosis, the exception being the presence of seminoma which improves it.

\section{Method}

All patients initially received a course of conventional therapy. Radical orchidectomy, where the testes and appendages are removed with an intact parietal Tunica vaginalis and spermatic cord to the internal ring was performed, the cord being occluded at the interior ring as a preliminary to the main dissection.

Postoperative radiotherapy was given by opposing fields at 200 rads mid-point tumour dose daily to 4600 rads on the $4 \mathrm{Me} \mathrm{V}$ Linear accelerator or Cobalt 60 units to the para-aortic and ipsilateral iliac lymph nodes. Treatment fields were defined using bi-pedal lymphography combined with intra-venous urography.

Chemotherapy was commenced 28 days after completion of irradiation. Eight patients received a combined regime of Bleomycin $15 \mathrm{mg} / \mathrm{M}^{2} /$ body surface area intra-muscularly twice weekly to a total dose of $400 \mathrm{mg}$, combined with vinblastine intra-venously on days one and two of a 21 day cycle. Initially the dose of vinblas- 
tine used was $0.13 \mathrm{mg} / \mathrm{kg}$ body weight but after unacceptable side effects (vide infra) this was reduced by $50 \%$. Vinblastine was continued for one year in patients remaining disease free. One patient received vinchristine alone $1 \mathrm{mg}$ every day 21 days for a year.

Follow-up was carried out every six weeks by clinical examination, chest X-ray, and full blood counts. Interval assays of Alpha-feto-protein, and Beta human chorionic gonado trophin were performed.

\section{Results}

These are detailed in Table I. Three patients have died, two within a year of orchidectomy, with disease free intervals of four, six and nine months (but retrospective analysis of symptoms in the last case suggests this should be considered much shorter).

Side effects

Patient No 2 developed severe marrow depression with a total white cell count of less than $0.6 \times 10^{9} / 1$, and this effect was noted in two other patients with Stage III disease being treated concurrently, and occurred maximally 10 days after first dose of vinblastine. Patient No 1 maintained a satisfactory total white cell count above $2.5 \times: 10^{\circ} / 1$ but suffered severe malaise whilst vinblastine therapy was maintained. Following these experiences the dose of vinblastine was reduced by $50 \%$ and side effects on this altered regime were few. Total white cell counts fell to just below $3.0 \times 10^{9} / 1$ after the first dose of vinblastine, and two patients developed severe nausea and vomiting whilst receiving both drugs. Three patients developed skin thickening over elbows, knees and hands as has been reported in patients receiving high dose Bleomycin, by Spige ${ }^{10}$, but these resolved when the drug was discontinued on reaching the $400 \mathrm{mg}$ dose. No pulmonary lesions of the type ascribed to Bleomycin by Blum ${ }^{11}$ were seen. Those patients who have survived to date returned to their duties (albeit restricted from hard physical work, long hours and exposure to climatic extremes) on completion of their Bleomycin. One patient had a significant hair loss which recovered on completion of treatment.

\section{Discussion}

Testicular teratoma are essentially cancers of the younger age groups maximum incidence being in the third and fourth decades and form the leading cause of death from malignancy in males between 25 and 35 years (Williams ${ }^{12}$ ). With conventional therapy alone survival rates are poor, medium three year survival rates being less than $50 \%$ for some histological types overall $\left(\mathrm{Pugh}^{8}\right)$, and Field ${ }^{5}$ found this to be as low as $40 \%$ at two years in our own British Army series. There is great variation from one series to another, for example the London Hospital series found MTU Stage I to have a 35\% three year survival ${ }^{13}$ whilst Manchester series claim $71 \%{ }^{14}$. These discrepancies probably reflect the difficulty in histological grouping and the small numbers available.

Histological type would appear to be of such profound importance that we believe each type should be considered as a separate disease entity, and accordingly report our results based on this. We doubt whether any value can be obtained by 
Table I

Results of nine cases

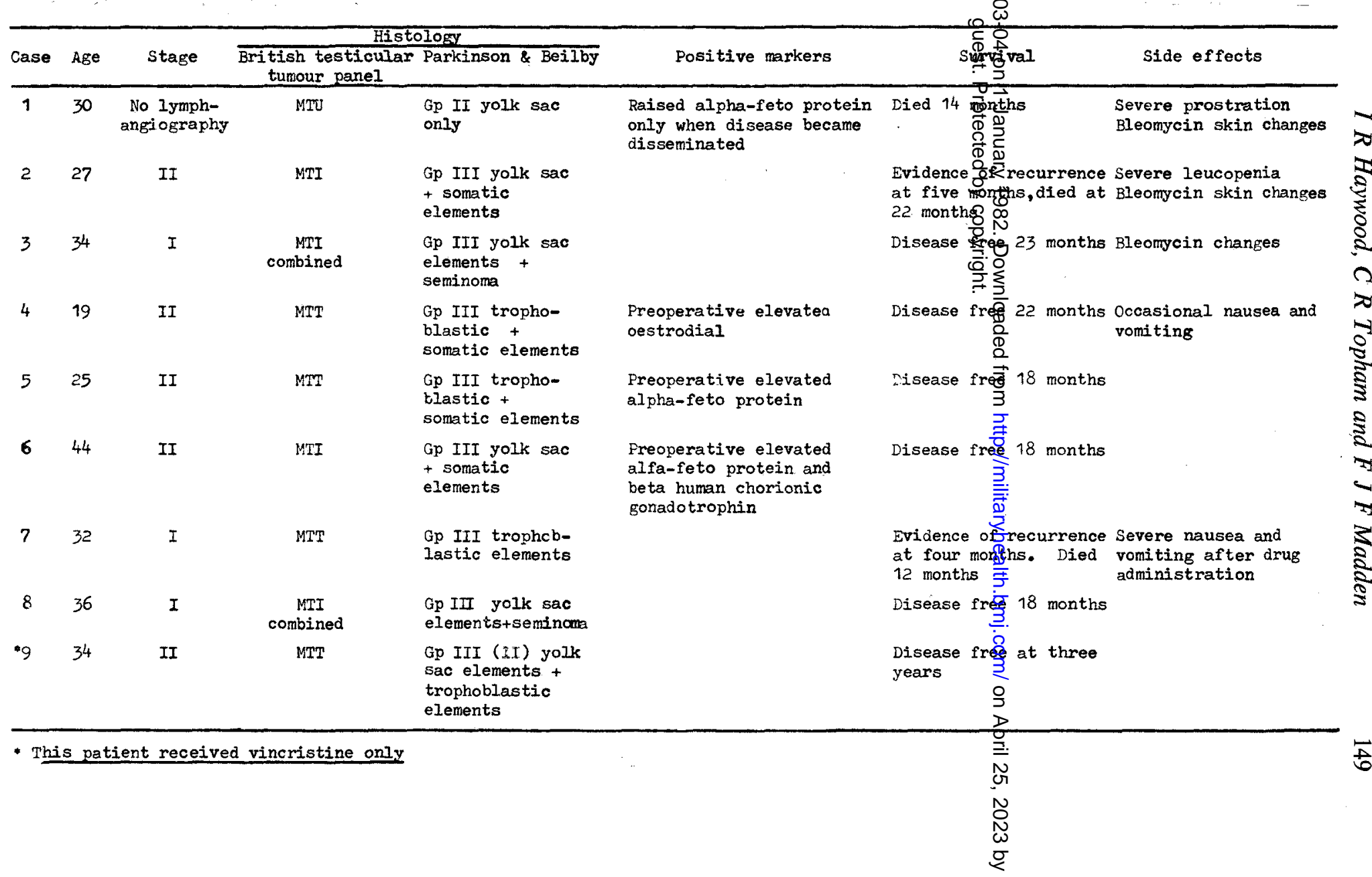


reviewing all types together as by Hoffken and Schmidt ${ }^{15}$ in their report on adjuvant therapy in Stage II disease. These tumours are not common and when divided into their many histological types, the incidence of some types becomes so rare that no centres are or will be able to collect sufficient numbers to make a statistically valid prospective trial feasible. We note that reports of percentage mortality in some series are based on one or two cases.

Of our surviving six patients two were Stage I tumours with mixed yolk sac and seminoma elements: a favourable histological type according to Parkinson ${ }^{9}$ and have disease free intervals of 18 and 23 months. The remaining four patients were all Stage II, three being mixed trophoblastic and somatic elements with disease free intervals of 18 months, and the fourth which contained mixed yolk sac and trophoblastic elements implying a particularly poor prognosis, so far has a disease free interval of three years.

Review of the three deaths is perhaps of more value. One was Stage II, one could not be staged since lymphography was not performed, and the third although Stage I histologically contained large quantities of trophoblastic elements. All three had very short intervals between orchidectomy and the appearance of distant metastases, suggesting rapidly dividing tumours in which by the time chemotherapy had been commenced (approximately eleven weeks after orchidectomy), distant metastic cell mass was beyond its scope. Chemo-sensitivity must, however, be of some importance since remissions are reported when a tumour mass was obviously in excess of that in these cases and our own observations of Stage III patients confirm this. The possibility remains that commencement of chemotherapy earlier might have been of value.

Samuels ${ }^{16}$ noted that vinblastine induced leucopenia was more severe in those of their patients who had been irradiated, and we conclude that the irradiation in our patients was instrumental in the marrow depression which forced us to reduce the dose. However, we were impressed by the tolerance of the patients to the low dose, an important concept when using chemotherapy in the adjuvant situation in contrast to the therapy of a patient with established disease.

The twin problems of delay between orchidectomy and commencement of chemotherapy, and the restriction of dosage lead to re-appraisal of the role of radiotherapy. In many centres in North America, the European continent and at least one in the United Kingdom, the retroperitoneal lymph nodes are treated by radical block dissection. This has the advantage of being able to use larger dose regimes as rocommended by Hoffken ${ }^{15}$, and also allows more accurate staging of the disease. Surgically this is a formidable procedure in which $11 \%$ are said to develop major complications (Williams ${ }^{12}$ ). However, mega-voltage irradiation to the paraaortic region is not without morbidity. Roswit ${ }^{17}$ found that in a long 10 year followup of 256 American soldiers who had received para-aortic irradiation for testicular neoplasms, $14 \%$ developed disabling upper abdominal complications. Admittedly many of these had received a total tumour dose in excess of 4500 rads, but even in those who received between 3500 to 4500 rads $24 \%$ developed a non-ulcerative dyspepsia, and 5\% small bowel problems. We have not seen anything like this number in over 400 cases treated in this unit. The control rate of the disease by 
retro-peritoneal block dissection and by irradiation appears to be about the same (Smithers ${ }^{18}$ ), but when nodes are greater than $2 \mathrm{~cm}$ radiation is less effective (Tyrell $^{19}$ ). An alternative approach might be to administer several courses of chemotherapy between orchidectomy and radiotherapy. This would overcome both problems of delay and dose restriction, and possibly reduce any large retro-peritoneal nodes to within the $2 \mathrm{~cm}$ critique, thus improving the likelihood of tumour sterilisation by irradiation.

In conclusion we feel there is a case for early adjuvant chemotherapy in the less favourable histological types, for example, those containing yolk sac and trophoblastic elements (Parkinson and Beilby, Group III). This is supported by the limited figures available from the London Hospital and the Manchester series, and by our failure to control the disease in those patients who died.

\section{Acknowledgements}

We should like to thank Maj Gen A P Dignan, Cols E R Briant and P W B Scott, and Dr K A Newton for their help and encouragement.

\section{REFERENCES}

1. STEPHEN R A. Malignant testicular tumours. Aberdeen 1960.

2. FIELD T E. Testicular tumours in army patients. Royal Army Medical College Library 1963.

3. Einhorn L H and DONonue I P. Improved chemotherapy in disseminated testicular cancer. J Urol 1977; 117: 65-72.

4. Samuels M L, Holoye $P$ Y and Johnson D E. Bleomycin combination chemotherapy in the management of testicular neoplasia. Cancer 1975; 36: 318-326.

5. Field T. E. Common errors occurring in the diagnosis of testicular neoplasms and the effect of these errors or prognosis. J R Army Med Corps 1964; 110: 152-155.

6. Staubitz W J and Magoss I V. Surgical management of testis tumours. J Urol 1969; 101: $350-364$.

7. MAIER J G and SULAK M H. Radiation therapy in malignant testicular tumours. Cancer 1973; 32: 1217-1226.

8. Pugh R C B. Pathology of the Testes. London. Blackwell Scientific Publications 1976.

9. Parkinson $\mathrm{C}$ and BeILbY $\mathrm{J} O \mathrm{O}$. Features of prognostic significance in testicular germ cell tumours. J Clin Pathol 1977; 30: 113-119.

10. SPIGel S C and Coltman C A. Vinblastine and Bleomycin therapy for disseminated testicular tumours. Cancer Chemotherapy Reports 1974; 58: 213-216.

11. Blum R H, Carter S K and AGRe K. A clinical review of Bleomycin. Cancer 1973; 31: $903-914$.

12. Williams $C$. Current dilemmas in the management of non-seminomatous germ cell tumours of the testes. Cancer Treat Rep 1977; 4.

13. Blandy J P. Testicular Tumours. Recent Advances in Surgery No 9. Ed Selwyn Taylor. London. Churchill Livingstone 1977: 249-268.

14. Dische $\mathbf{S} 1978$ (personal communication).

15. HoFfKen $\mathbf{K}$ and SchmidT $\mathbf{C}$ G. Chemotherapy in Stage II testicular tumours. Z Krebsforsch $1976 ; 86$ : 103-108.

16. SAMUels M L, JohNSON D E and Holoye P Y. Bleomycin combination chemotherapy in, Stage III Testicular Neoplasia. Gann Monography on Cancer Research 1976; 19: 269-275.

17. Roswit B, Malsky S J and ReID C B. Severe radiation injuries of the stomach, small bowel, colon and rectum. Am J Roentgenol 1972; 114: 460-475.

18. SMIthers SiR D W. The Management of the Patient with Tumour of the Testis. In Pathology of the Testes. Ed R C B Pugh. London Blackwell Scientific Publications.

19. Tyrell $\mathrm{C} J$ and Peckham $M$ J. The response of lymph node metastases in testicular teratoma to radiation therapy. Br J Urol 1976; 48: 363 368. 